\title{
N-alkylation methods, Characterization and Evaluation of antibacterial activity of some Novel 5-Chloroisatin Derivatives
}

\author{
Z. Tribak ${ }^{1}$, O.El Amin ${ }^{2}$, M.K. Skalli ${ }^{1 *}$, O. Senhaji ${ }^{3}$, Y. Kandri. Rodi ${ }^{4}$, \\ M. Houssaini. Iraqui ${ }^{2}$ \\ ${ }^{1}$ Laboratory of Applied Chemistry, Sidi Mohamed Ben Abdellah University, Faculty of Sciences and Technology \\ of Fes, Morocco \\ ${ }^{2}$ Laboratory of Microbial Biotechnology, Faculty of Science and Technology, B.P. 2202, Fes, Morocco \\ ${ }^{3}$ Laboratory of Applied Physical Chemistry, Moulay Ismail University, Faculty of Sciences and Technology of \\ Errachidia, Morocco. \\ ${ }^{4}$ Laboratory of Applied Organic Chemistry, Sidi Mohamed Ben Abdellah University, Faculty of Science and \\ Technology of Fes, Morocco.
}

\begin{abstract}
:
A series of new 5-Chloroisatin derivates have been synthesized by the method of $\mathrm{N}$-alkylation at room temperature, in the presence of a base and a catalyst with good yields. The chemical structures of these compounds were confirmed by NMR $\left({ }^{1} \mathrm{H} \&{ }^{13} \mathrm{C}\right)$, these new compounds obtained were evaluated for their antibacterial activity. The final results revealed that the majority of the compounds exhibited good antimicrobial activity against various organisms.
\end{abstract}

Keywords: 5-Chloroisatin derivates, $\mathrm{N}$-alkylation, antibacterial activity, NMR

\section{INTRODUCTION}

The design, synthesis and production of molecules, are ones of the important objectives of organic and medicinal chemistry [1], which are having highly therapeutic interest. Since the discovery of heterocyclic nucleus the chemistry of isatin and their fused derivatives has a wide varity of pharmacological activities such as antimicrobial, anticancer, antiviral, anticonvulsant, antiinflammatory and analgesic [2]. Different research group attempted study on isatin synthetic aspect. Otherresearch group attempted study of isatin biological activity [3].

Thus the structural modification of the isatin derivates moiety is still of the highest importance. This article has focused on the application of synthetic $\mathrm{N}$-alkylation methods in the conditions of charge transfer catalysis in the presence of a base $\left(\mathrm{K}_{2} \mathrm{CO}_{3}\right)$ and a catalyst (BTBA) between 5-Chloro- $1 H$-indole-2,3-dione and alkylating agents with good to excellent yields [4]. The synthesized 5-Chloroisatin derivatives were characterized by spectroscopic techniques, such as, ${ }^{1} \mathrm{H}$ NMR, ${ }^{13} \mathrm{C}$ NMR. In addition, they were evaluated for their biological activities [5] as antibacterial in vitro against two Gram positive bacteria such as Staphylococcus aureus, Bacillus cereus and two Gram negative bacteria such as Escherichia coli, pseudomonas aeruginosa.

\section{MATERIAL AND METHODS}

\section{Chemistry:}

All melting points were measured by the Kofler bench. The ${ }^{1} \mathrm{H}$ NMR, ${ }^{13} \mathrm{C}$ NMR spectra were recorded on Bruker 300 NMR spectrometer advancement in $\mathrm{CDCl}_{3}$ using tetramethylsilane (TMS) as reference. Chemical shifts are reported in parts per million. Reactions were monitored by thin layer chromatography (TLC) on silica gel, plates were visualizing with ultraviolet light or iodine. Column chromatography was performed on silica gel $60 \quad(0.043-0.06 \mathrm{~mm}) \quad$ Merck.5-Chloroisatin derivatives were synthesized by alkylation method available in the phase transfer catalysis conditions [6, 7]. The synthetic strategies adopted to obtain target compounds are depicted in Figure 1 and 2.

\subsection{GENERAL PROCEDURE FOR THE SYNTHESIS OF NEW 5-CHLOROISATIN DERIVATES:}

Compounds were then reacted with various alkylating agent, so into a $100 \mathrm{~mL}$ two-necked flask we introduce $0.2 \mathrm{~g}(1.1 \mathrm{mmol})$ of 5-chloro- $1 \mathrm{H}$ indole-2,3-dione $(0.23 \mathrm{~g}, 1.16 \mathrm{mmol})$ of potassium carbonate in $15 \mathrm{ml}$ of $\mathrm{N}$-N-dimethylformamide (DMF) and $(0.035 \mathrm{~g}, 0.10 \mathrm{mmol})$ of BTBA with magnetic stirring, the alkylating agent is added slowly, the mixture is left at room temperature for 48 hours. During this period the progress of the reaction is monitored by TLC (thin layer chromatography). Once the reaction is complete, the salts are removed 
by filtration, the solvent (DMF) is evaporated under reduced pressure. The product obtained is purified on a column of silica gel eluent (ethyl acetate / hexane)

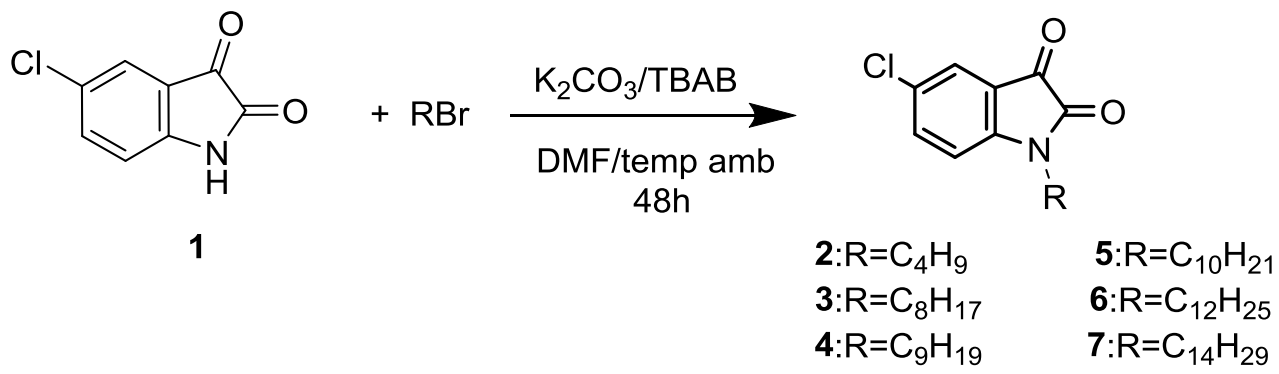

Figure 1: Synthesis of new products from 5-chloro- $1 H$-indole -2,3-dione<smiles>O=C1Nc2ccc(Cl)cc2C1=[OH+]</smiles>

1

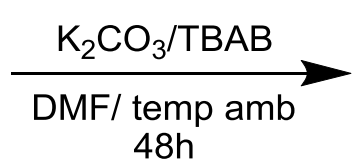

$48 \mathrm{~h}$<smiles>O=C1C(=O)N(Br)c2ccc(Cl)cc21</smiles>

$8: \mathrm{R}=\mathrm{C}_{6} \mathrm{H}_{12}$

Figure 2: Synthesis of novel derivatives of 5-Chloro- $1 H$-indole -2,3-dione by catalysis by phase transfer

\subsection{SPECTRAL DATA}

Compound 2 :1-butyl-5-chloroindoline-2,3-dione: Yield: $85 \%$; m.p: $80-82{ }^{\circ} \mathrm{C} ; \mathrm{R}_{\mathrm{f}}=0.85 ;{ }^{1} \mathrm{H}$ NMR $\left(\mathrm{CDCl}_{3} ; 300 \mathrm{MHz}\right): \delta(\mathrm{ppm})$ 7.54-7.55 (d, H, $\left.\mathrm{H}_{\mathrm{Ar}},{ }^{4} \mathrm{~J}_{\mathrm{H}-\mathrm{H}}=3 \mathrm{~Hz}\right) 7.507 .51\left(\mathrm{~d}, \mathrm{H}, \mathrm{H}_{\mathrm{Ar}},{ }^{4} \mathrm{~J}_{\mathrm{H}-\mathrm{H}}=3 \mathrm{~Hz}\right)$; $6.84\left(\mathrm{~d}, \mathrm{H}, \mathrm{H}_{\mathrm{Ar} ;}{ }^{3} \mathrm{~J}_{\mathrm{H}-\mathrm{H}}=6 \mathrm{~Hz}\right) ; 3.72\left(\mathrm{t}, 2 \mathrm{H}, \mathrm{CH}_{2},{ }^{3} \mathrm{~J}_{\mathrm{H}-\mathrm{H}}\right.$ $=6 \mathrm{~Hz}) ; 1.60-1.70\left(\mathrm{~m}, 2 \mathrm{H}, \mathrm{CH}_{2}\right) ; 1.35-1.42(\mathrm{~m}$, $\left.2 \mathrm{H}, \mathrm{CH}_{2}\right), 0.95\left(\mathrm{t}, 3 \mathrm{H}, \mathrm{CH}_{3},{ }^{3} \mathrm{~J}_{\mathrm{H}-\mathrm{H}}=9 \mathrm{~Hz}\right) .{ }^{13} \mathrm{C} \mathrm{NMR}$ $\left(\mathrm{CDCl}_{3} ; 75 \mathrm{MHz}\right): \delta(\mathrm{ppm}) 183.83(\mathrm{C}=\mathrm{O}) ; 163.28$ $(\mathrm{N}-\mathrm{C}=\mathrm{O}) ; 142.43,136.12,121.06(\mathrm{Cq}) ; 137.61$, 125.38, $111.40\left(\mathrm{CH}_{\mathrm{Ar}}\right)$; 40.16, 29.24, $20.14\left(\mathrm{CH}_{2}\right)$; $13.67\left(\mathrm{CH}_{3}\right)$.

Compound 3 : 5-chloro-1-octylindoline-2,3-dione: Yield: $88 \%$; m.p: $68-70^{\circ} \mathrm{C} ; \mathrm{R}_{\mathrm{f}}=0.85 ;{ }^{1} \mathrm{H}$ NMR $\left(\mathrm{CDCl}_{3} ; 300 \mathrm{MHz}\right): \delta(\mathrm{ppm}) 7.54-7.55\left(\mathrm{~d}, \mathrm{H}, \mathrm{H}_{\mathrm{Ar}},{ }^{4} \mathrm{~J}_{\mathrm{H}-}\right.$ $\mathrm{H}=3 \mathrm{~Hz}) ; 7.50-7.51\left(\mathrm{~d}, \mathrm{H}, \mathrm{H}_{\mathrm{Ar}},{ }^{4} \mathrm{~J}_{\mathrm{H}-\mathrm{H}}=3 \mathrm{~Hz}\right) ; 6.84(\mathrm{~d}$, $\left.\mathrm{H}, \mathrm{H}_{\mathrm{Ar}},{ }^{3} \mathrm{~J}_{\mathrm{H}-\mathrm{H}}=9 \mathrm{~Hz}\right) ; 3.68\left(\mathrm{t}, 2 \mathrm{H}, \mathrm{CH}_{2},{ }^{3} \mathrm{~J}_{\mathrm{H}-\mathrm{H}}=6 \mathrm{~Hz}\right)$; 1.71-1.53 (m, $\left.2 \mathrm{H}, \mathrm{CH}_{2}\right) ; 1.24-1.31\left(\mathrm{~m}, 10 \mathrm{H}, \mathrm{CH}_{2}\right)$, $0.85\left(\mathrm{t}, 3 \mathrm{H}, \mathrm{CH}_{3},{ }^{3} \mathrm{~J}_{\mathrm{H}-\mathrm{H}}=6 \mathrm{~Hz}\right),{ }^{13} \mathrm{C} \mathrm{NMR}\left(\mathrm{CDCl}_{3}\right.$; $75 \mathrm{MHz}): \delta(\mathrm{ppm}) 183.20(\mathrm{C}=\mathrm{O}) ; 160.73(\mathrm{~N}-\mathrm{C}=\mathrm{O})$; 144.60, 137.60, 125.37 (Cq); 129.45, 115.79, 111.42 $\left(\mathrm{CH}_{\mathrm{Ar}}\right) ; \quad 40.47, \quad 31.73, \quad 29.19, \quad 27.20, \quad 26.88$, $22.60\left(\mathrm{CH}_{2}\right) ; 14.04\left(\mathrm{CH}_{3}\right)$.

Compound 4: 5-chloro-1-nonylindoline-2,3dione: Yield: $87 \%$; m.p: $67-68{ }^{\circ} \mathrm{C} ; \mathrm{R}_{\mathrm{f}}=0 . .82 ;{ }^{1} \mathrm{H}$ NMR $\left(\mathrm{CDCl}_{3} ; 300 \mathrm{MHz}\right): \delta(\mathrm{ppm}) 7.58-7.59(\mathrm{~d}, \mathrm{H}$, $\left.\mathrm{H}_{\mathrm{Ar}},{ }^{4} \mathrm{~J}_{\mathrm{H}-\mathrm{H}}=3 \mathrm{~Hz}\right) ; 7.54-7.55\left(\mathrm{~d}, \mathrm{H}, \mathrm{H}_{\mathrm{Ar}},{ }^{4} \mathrm{~J}_{\mathrm{H}-\mathrm{H}}=3 \mathrm{~Hz}\right)$; $6.85\left(\mathrm{~d}, \mathrm{H}, \mathrm{H}_{\mathrm{Ar}},{ }^{3} \mathrm{~J}_{\mathrm{H}-\mathrm{H}}=9 \mathrm{~Hz}\right) ; 3.70\left(\mathrm{t}, 2 \mathrm{H}, \mathrm{CH}_{2},{ }^{3} \mathrm{~J}_{\mathrm{H}-\mathrm{H}}\right.$ $=9 \mathrm{~Hz}) ; 1.65-1.75\left(\mathrm{~m}, 2 \mathrm{H}, \mathrm{CH}_{2}\right) ; 1.28\left(\mathrm{~m}, 12 \mathrm{H}, \mathrm{CH}_{2}\right)$, $0.89\left(\mathrm{t}, 3 \mathrm{H}, \mathrm{CH}_{3},{ }^{3} \mathrm{~J}_{\mathrm{H}-\mathrm{H}}=6 \mathrm{~Hz}\right) .{ }^{13} \mathrm{C} \mathrm{NMR}\left(\mathrm{CDCl}_{3}\right.$; 75MHz): $\delta(\mathrm{ppm}) 181.84(\mathrm{C}=\mathrm{O}) ; 167.00(\mathrm{~N}-\mathrm{C}=\mathrm{O})$;
147.07, 137.63, 129.39 (Cq); 141.28, 116.10, 110.29 $\left(\mathrm{CH}_{\mathrm{Ar}}\right)$; 42.53, 32.42, 29.49, 29.27, 29.21, 27.21, 26.89, $23.18\left(\mathrm{CH}_{2}\right) ; 15.83\left(\mathrm{CH}_{3}\right)$.

Compound 5: 5-chloro-1-decylindoline-2,3-dione: Yield: $86 \%$; m.p: $62-64^{\circ} \mathrm{C} ; \mathrm{R}_{\mathrm{f}}=0.77 ;{ }^{1} \mathrm{H}$ NMR $\left(\mathrm{CDCl}_{3} ; 300 \mathrm{MHz}\right): \delta(\mathrm{ppm}) 7.53\left(\mathrm{~d}, \mathrm{H}, \mathrm{H}_{\mathrm{Ar}},{ }^{4} \mathrm{~J}_{\mathrm{H}-\mathrm{H}}\right.$ $=3 \mathrm{~Hz}) ; 7.50\left(\mathrm{~d}, \mathrm{H}, \mathrm{H}_{\mathrm{Ar}}, \mathrm{J}_{\mathrm{H}-\mathrm{H}}=3 \mathrm{~Hz}\right) ; 6.80(\mathrm{~d}, \mathrm{H}$, $\left.\mathrm{H}_{\mathrm{Ar}},{ }^{3} \mathrm{~J}_{\mathrm{H}-\mathrm{H}}=9 \mathrm{~Hz}\right) ; 3.67\left(\mathrm{t}, 2 \mathrm{H}, \mathrm{CH}_{2},{ }^{3} \mathrm{~J}_{\mathrm{H}-\mathrm{H}}=6 \mathrm{~Hz}\right)$; 1.60-1.67 (m, 2H, $\left.\mathrm{CH}_{2}\right) ; 1.22\left(\mathrm{~m}, 14 \mathrm{H}, \mathrm{CH}_{2}\right), 0.84$ $\left(\mathrm{t}, 3 \mathrm{H}, \quad \mathrm{CH}_{3},{ }^{3} \mathrm{~J}_{\mathrm{H}-\mathrm{H}}=6 \mathrm{~Hz}\right) .{ }^{13} \mathrm{C} \mathrm{NMR}\left(\mathrm{CDCl}_{3}\right.$; $75 \mathrm{MHz}): \delta(\mathrm{ppm}) 183.15(\mathrm{C}=\mathrm{O}) ; 166.03(\mathrm{~N}-\mathrm{C}=\mathrm{O})$; $145.52,129.39,125.39(\mathrm{Cq}) ; 141.28,115.58,111.40$ $\left(\mathrm{CH}_{\mathrm{Ar}}\right)$; 40.49, 31.87, 29.49, 29.27, 29.21, 27.21, 26.89, $22.70\left(\mathrm{CH}_{2}\right) ; 14.12\left(\mathrm{CH}_{3}\right)$.

Compound 6 :5-chloro-1-dodecylindoline-2,3dione: Yield: $85 \%$; m.p: $64-66{ }^{\circ} \mathrm{C} ; \mathrm{R}_{\mathrm{f}}=0.75 ;{ }^{1} \mathrm{H}$ NMR $\left(\mathrm{CDCl}_{3} ; 300 \mathrm{MHz}\right): \delta(\mathrm{ppm}) 7.55(\mathrm{~d}, \mathrm{H}$, $\left.\mathrm{H}_{\mathrm{Ar}},{ }^{4} \mathrm{~J}_{\mathrm{H}-\mathrm{H}}=3 \mathrm{~Hz}\right) ; 7.51\left(\mathrm{~d}, \mathrm{H}, \mathrm{H}_{\mathrm{Ar}},{ }^{4} \mathrm{~J}_{\mathrm{H}-\mathrm{H}}=3 \mathrm{~Hz}\right) ; 6.82$ $\left(\mathrm{d}, \mathrm{H}, \mathrm{H}_{\mathrm{Ar}}{ }^{3} \mathrm{~J}_{\mathrm{H}-\mathrm{H}}=9 \mathrm{~Hz}\right) ; 3.69\left(\mathrm{t}, 2 \mathrm{H}, \mathrm{CH}_{2},{ }^{3} \mathrm{~J}_{\mathrm{H}-\mathrm{H}}\right.$ $=9 \mathrm{~Hz}) ; 1.61-1.70\left(\mathrm{~m}, 2 \mathrm{H}, \mathrm{CH}_{2}\right) ; 1.23\left(\mathrm{~s}, 18 \mathrm{H}, \mathrm{CH}_{2}\right)$, 0.86(t, $\left.3 \mathrm{H}, \quad \mathrm{CH}_{3}, \quad{ }^{3} \mathrm{~J}_{\mathrm{H}-\mathrm{H}} \quad=6 \mathrm{~Hz}\right) . \quad{ }^{13} \mathrm{C} \quad \mathrm{NMR}$ $\left(\mathrm{CDCl}_{3} ; 75 \mathrm{MHz}\right): \delta(\mathrm{ppm}) 184.39(\mathrm{C}=\mathrm{O}) ; 162.95$ $(\mathrm{N}-\mathrm{C}=\mathrm{O}) ; 149.63, \quad 129.47,118.17(\mathrm{Cq}) ; 137.64$, 125.38, $111.44\left(\mathrm{CH}_{\mathrm{Ar}}\right)$; 40.49, 31.93, 29.61, 29.45, 29.46, 29.34, 29.21, 27.21, 26.89, $22.70\left(\mathrm{CH}_{2}\right)$; $14.13\left(\mathrm{CH}_{3}\right)$.

Compound 7 : 5-chloro-1-tetradecylindoline-2,3dione: Yield: $84 \%$; m.p : $65-67^{\circ} \mathrm{C} ; \mathrm{R}_{\mathrm{f}}=0.73 ;{ }^{1} \mathrm{H}$ NMR $\left(\mathrm{CDCl}_{3} ; 300 \mathrm{MHz}\right): \delta$ (ppm) $7.54(\mathrm{~d}, \mathrm{H}$, $\left.\mathrm{H}_{\mathrm{Ar}},{ }^{4} \mathrm{~J}_{\mathrm{H}-\mathrm{H}}=3 \mathrm{~Hz}\right) ; 7.50\left(\mathrm{~d}, \mathrm{H}, \mathrm{H}_{\mathrm{Ar}},{ }^{4} \mathrm{~J}_{\mathrm{H}-\mathrm{H}}=2.1 \mathrm{~Hz}\right) ; 6.84$ $\left(\mathrm{d}, \mathrm{H}, \mathrm{H}_{\mathrm{Ar}},{ }^{3} \mathrm{~J}_{\mathrm{H}-\mathrm{H}}=9 \mathrm{~Hz}\right) ; 3.68\left(\mathrm{t}, 2 \mathrm{H}, \mathrm{CH}_{2},{ }^{3} \mathrm{~J}_{\mathrm{H}-\mathrm{H}}\right.$ 
$=9 \mathrm{~Hz}) ; 1.60-1.70\left(\mathrm{~m}, 2 \mathrm{H}, \mathrm{CH}_{2}\right) ; 1.22\left(\mathrm{~s}, 22 \mathrm{H}, \mathrm{CH}_{2}\right)$, $0.85\left(\mathrm{t}, 3 \mathrm{H}, \mathrm{CH}_{3},{ }^{3} \mathrm{~J}_{\mathrm{H}-\mathrm{H}}=6 \mathrm{~Hz}\right) .{ }^{13} \mathrm{C} \mathrm{NMR}\left(\mathrm{CDCl}_{3}\right.$; $75 \mathrm{MHz}): \delta(\mathrm{ppm}) 182.24(\mathrm{C}=\mathrm{O}) ; 161.85(\mathrm{~N}-\mathrm{C}=\mathrm{O})$; $137.62,125.35,111.41(\mathrm{Cq}) ; 130.45,129.43,118.46$ $\left(\mathrm{CH}_{\mathrm{Ar}}\right)$; 40.48, 31.94, 29.65, 29.62, 29.61, 29.54, $29.46,29.36,29.21,27.20,26.88,22.70\left(\mathrm{CH}_{2}\right)$; $14.12\left(\mathrm{CH}_{3}\right)$.

Compound 8: 1-(6-bromohexyl)-5chloroindoline-2,3-dione:Yield: $85 \%$; m.p: 66$70{ }^{\circ} \mathrm{C} ; \mathrm{R}_{\mathrm{f}}=0.8$ (Hexane/EtOAc, $2: 1$ ); ${ }^{1} \mathrm{H}$ NMR $\left(\mathrm{CDCl}_{3} ; 300 \mathrm{MHz}\right): \delta(\mathrm{ppm})$ 7.49-7.51 (dd, H, $\left.\mathrm{CH}_{\text {Arom }}, \mathrm{J}_{\mathrm{H}-\mathrm{H}}^{4}=1.5 \mathrm{~Hz}, \mathrm{~J}_{\mathrm{H}-\mathrm{H}}^{3}=4.5 \mathrm{~Hz}\right) ; 7.47(\mathrm{~d}, \mathrm{H}$, $\left.\mathrm{CH}_{\mathrm{Ar}}, \mathrm{J}_{\mathrm{H}-\mathrm{H}}^{4}=1.5 \mathrm{~Hz}\right) ; 6.77\left(\mathrm{~d}, \mathrm{H}, \mathrm{CH}_{\text {Arom }}, \mathrm{J}_{\mathrm{H}-\mathrm{H}}^{3}=6\right.$ $\mathrm{Hz}) ; 3.65\left(\mathrm{t}, 2 \mathrm{H}, \mathrm{CH}_{2}, \mathrm{~J}_{\mathrm{H}-\mathrm{H}}^{4}=3 \mathrm{~Hz}\right) ; 3.33(\mathrm{t}, 2 \mathrm{H}$, $\left.\mathrm{CH}_{2}, \mathrm{~J}_{\mathrm{H}-\mathrm{H}}^{3}=6 \mathrm{~Hz}\right) ; 1.75-1.82\left(\mathrm{~m}, 2 \mathrm{H}, \mathrm{CH}_{2}\right), 1.61-$ $1.68\left(\mathrm{~m}, 2 \mathrm{H}, \mathrm{CH}_{2}\right), 1.47\left(\mathrm{~m}, 4 \mathrm{H}, \mathrm{CH}_{2}\right) .{ }^{13} \mathrm{C} \mathrm{NMR}$ $\left(\mathrm{CDCl}_{3} ; 75 \mathrm{MHz}\right): \delta(\mathrm{ppm}): 182.64(\mathrm{C}=\mathrm{O}) ; 160.28$ $(\mathrm{N}-\mathrm{C}=\mathrm{O}) ; 146.49,132.61,119.49(\mathrm{Cq}) ; 134.44$, $130.93,123.33\left(\mathrm{CH}_{\mathrm{Ar}}\right) ; 40.51,33.70,32.01,27.89$, 27.07, $26.15\left(\mathrm{CH}_{2}\right)$.

\section{IN VITRO ANTIBACTERIAL ACTIVITY EVALUATION}

\subsection{A disc diffusion test:}

This method makes it possible to evaluate the antibacterial activity of a product. Although it is recognized as reliable and reproducible, it is mostly used as a preliminary stage for further studies as it provides access to essentially qualitative results. The technique used is a modification of the method of Hayes and Markovic.

It consists of depositing a sterile disk imbibed by the test product on a bacterial mat at the very beginning of its growth and measuring the area where the bacteria could not develop. The inhibition diameter, which reflects the antibacterial activity of the product tested, is thus determined. The antimicrobial activity of 5-Chloro- $1 \mathrm{H}$-indole-2,3dione derivatives was evaluated using the disc diffusion method [8] using different microorganisms, including three types of bacteria: Bacillus cereus, Staphylococcus aureus, Escherichia coli and Pseudomonas aeruginosa. Mueller Hinton agar medium (MHA) was used for bacteria. Plates were preincubated at $37^{\circ} \mathrm{C}$ for $24 \mathrm{~h}$.

A sterile paper disk (6 $\mathrm{mm}$ in diameter) was placed on the surface of each agar plate and impregnated with $5 \mu \mathrm{L}$ of each solution of 5-chloroisatin derivatives at a final concentration of $10 \mathrm{mg} / \mathrm{ml}$. Then, the Petri dishes are incubated at $37{ }^{\circ} \mathrm{C}$ for 24 $\mathrm{h}$ for the bacteria. The diameters of the inhibition zones were measured in $\mathrm{mm}$ (including the diameter of the disk) with the caliper. A disc impregnated with $2 \%$ dimethylsulfoxide as a negative control was made. Each experiment was carried out in triplicate.

\subsection{MINIMUM INHIBITORY \\ CONCENTRATION DETERMINATION (MIC):}

The minimal inhibitory concentration (MIC) of our products was evaluated according to the microdilution method with some modifications. The minimum inhibitory concentration (MIC) was determined as the lowest concentration with no microbial growth compared to the positive control. DMSO (2\%) was used as a negative control.

Briefly, the dilution of the 5-chloroisatin derivatives was prepared in a Mueller Hinton broth supplemented with bacteriological agar, to reach a final concentration between $5 \mathrm{mg} / \mathrm{ml}$ and $0.004 \mathrm{mg}$ / $\mathrm{ml}, 50 \mu \mathrm{L}$ of bacterial inoculum was added to each well at a final concentration of $106 \mathrm{CFU} / \mathrm{ml}$.

The final concentration of our product was between $5 \mathrm{mg} \mathrm{ml}-1$ (3rd well) and $0.019 \mathrm{mg} \mathrm{ml}-1$ (well 11). Plates were incubated at $37^{\circ} \mathrm{C}$ for $24 \mathrm{~h}$. After 2 hours of a subsequent incubation, bacterial growth was revealed by reduction of blue dye resazurin to pink resorufin [9].

\subsection{MINIMUM BACTERICIDAL CONCENTRATIONS (MBC):}

order to determine the $\mathrm{CMB}$, a bactericidal control is carried out 24 hours earlier by streaking on a platelet agar, after microdilution to the broth by spreading $5 \mu$ of the negative wells on Luria Bertani agar plates (Luria Bertani medium: Yeast extract 5.0 $\mathrm{g}$, peptone $10.0 \mathrm{~g}$, sodium chloride $5.0 \mathrm{~g}$, distilled water $1000 \mathrm{ml}$ ). The $\mathrm{MBC}$ will be the lowest concentration whose transplant shows a growth of germ less than or equal to $0.01 \%$ of survivors [10].

\section{RESULTS AND DISCUSSION :}

Table 1: MICs and MBCs of the compounds against the microbes used

\begin{tabular}{ccccc}
\hline & \multicolumn{3}{c}{ MIC/MBC $\mathbf{( m g / m L ) ~}$} \\
\cline { 2 - 4 } compounds & Bacillus cereus & Staphylococcus & Escherichia coli & $\begin{array}{c}\text { Pseudomonas } \\
\text { aeruginosa }\end{array}$ \\
\cline { 2 - 5 } & & aureus & - \\
\hline $\mathbf{2}$ & $0,156 / 0,156$ & $0,313 / 0,313$ & - \\
\hline $\mathbf{3}$ & $0,078 / 0,078$ & $0,156 / 0,156$ & - \\
\hline $\mathbf{4}$ & $0,078 / 0,078$ & $0,01 / 0,01$ & - \\
\hline
\end{tabular}




\begin{tabular}{ccccc}
$\mathbf{6}$ & - & - & - & - \\
\hline $\mathbf{7}$ & - & - & - & - \\
\hline $\mathbf{8}$ & - & $1,25 / 1,25$ & $1,25 / 1,25$ &
\end{tabular}

This table showed that compound $\mathbf{4}$ exercised an excellent inhibitory activity against Staphylococcus aureus à Gram positive bacteria, with a MIC value of $0,01 \mathrm{mg} / \mathrm{mL}$, while compounds 3 and 4 displayed comparable activity against Bacillus cereus with a MIC value of $0,078 \mathrm{mg} / \mathrm{ml}$, while compounds $\mathbf{3}$ and $\mathbf{5}$ displayed moderate to good activity against Gram positive bacteria studied. The compounds 8 exhibited moderate inhibitory effect against Gram+ and Gram- bacteria (staphylococcus aureus and Escherichia coli) at a MIC value of 1,25 mg/mL. However, Pseudomonas aeruginosa (à Gram - bacteria) was resistant to alltested compounds. Moreover, the compounds 6 and 7 did not show any antimicrobial effects against all tested strains. Generally, Gram-positive bacteria are much more susceptible to antibacterial agents than Gram negative bacteria, whose resistance is attributed to the structures of their cell wall. Gramnegative bacteria have a thick lipid bilayer which is selectively permeable [11].

\section{CONCLUSION}

The synthesis of a variety of the 5Chloroisatin derivatives was carried out via the $\mathrm{N}$ alkylation reaction. All compounds were obtained in good yields. The structures of compounds obtained were confirmed by ${ }^{1}$ H-NMR, ${ }^{13}$ C-NMR. The antimicrobial activity of these compounds was tested against two Gram-negative bacteria and two Gram-positive bacteria. From the above results it can be concluded that, the highest activity was obtained with the $\mathbf{4}$ compound which exhibited good to high activity towards Staphylococcus aureus Gram positive bacteria, with a MIC value of 0.01 $\mathrm{mg} / \mathrm{ml}$. Compounds 2, 3, 5, 8 showed moderate activity against the Gram positive bacteria studied, whereas compounds $\mathbf{6}$ and $\mathbf{7}$ showed no antimicrobial effect.

\section{REFERENCES}

[1]. Z. Tribak, Y. Kandri.Rodi, A. Haoudi, E.M Essassi, F. Capet., H. Zouihrid, 1Allyl-5-chloroindoline-2,3dione.IUCrData (2016) 1, x160862

[2]. K. Meenakshi, N. Gopal, M. Sarangapani, synthesis, characterization and antimicrobial activity of some novel schiff and mannich bases of isatin Int. J. Pharm. Pharm Sci,6 (6) (2014), 318-322

[3]. S. N. Pandeya, Sriavastava, Anupam, Indole a versatile nucleus in pharmaceutical field Int. J. current Pharma. Review. Res Nov Jan 1 (2011) 31-17.

[4]. Z. Tribak, Y. Kandr. Rodi, Y. Kharbach, A. Haoudi, M.K. Skalli, A. Mazzah, M. Akhazzane. E.M. Essassi, synthesis of new 1H-indole-2,3-dione derivatives using phase-transfer catalysis and characterization by $x$-ray crystallography

J.Mar.Chim.Heterocycl. 15 (2016) 7984

[5]. S. Nareshvarma. P Satya.Shrivastava, P. Somarouthu. Synthesis and antimicrobial activity of some novel fused heterocyclic moieties $\mathrm{Org}$. Commun. 6 (2) (2013) 78-85

[6]. Z Tribak. YKandri. Rodi, A. Haoudi, E M. Essassi, F Capetc .H Zouihri, 1-(12Bromododecyl)-5-chloroindoline-2,3dione IUCrData (2016). 1, x160971

[7]. Z Tribak.Y Kandri. Rodi, A. Haoudi, E. M. Essassi, F Capetc, H. Zouihri, 1Benzyl-5-chloroindoline-2,3-dione. IUCrData (2016). 1, x160854

[8]. M. Balouiri, M. Sadiki, S.K Ibnsoud, Methods for in vitro evaluating antimicrobial activity: A reviewInter. $J$. Pharmacy. Pharmaceutical. Sc. 6 (2014) 6-9.

[9]. H. Ismaili, L. Milella, S. Fkih-Tetouani, A. Ilidrissi, A. Camporese, S. Sosa, G. Altinier, R.D Loggia, R Aquino. In vivo topical anti-inflammatory and in vitro antioxidant activities of two extracts of Thymus satureioides leaves 91 (2004) 31-36

[10]. S. Bouhdid, J. Abrini, A. Zhiri, M.J. Espuny, A. Manresa. Investigation of functional and morphological changes in Pseudomonas aeruginosa and Staphylococcus aureus cells induced by Origanum compactum essential oil $J$. Appl Microbiol, 106 (2009) 1558-1568

[11]. M. Blessi. J Maharaj, G. Krishnaveni G, M Brahmeshwari. G. Sarangapani. Sammaiah. Synthesis and Antimicrobial activity of some new Isatin derivatives. $J$ Advan Pharmaceu Sci 2011;1(1):20-33 